\title{
As percepções do comportamento de consumo ecológico de estudantes universitários
}

\author{
Allen Krysthiano Saraiva Figueiredo ${ }^{1}$ \\ Maicon Richard Tabelini ${ }^{2}$
}

\begin{abstract}
Resumo: A presente investigação teve como objetivo identificar a percepção do comportamento de consumo ecologicamente consciente (CCEC) de estudantes universitários, por meio da readaptação da pesquisa de Yeh (2015), trabalho que foi inspirado e adaptado do estudo de Zhao et al. (2014). O referencial teórico aborda as temáticas do marketing verde, comportamento de consumo ecologicamente consciente, estudos correlatos sobre a estrutura de comportamentos socialmente responsáveis do consumidor entre atitudes e comportamentos favoráveis ao meio ambiente; e a sistematização de variáveis comportamentais, motivadores internos e externos que exercem impacto sobre o CCEC. Realizou-se uma pesquisa quantitativa e descritiva. Os dados foram coletados mediante a aplicação de questionário online direcionado para estudantes universitários dos Estados da Bahia e Espírito Santo. O instrumento de pesquisa foi composto de 32 afirmativas estruturadas com os construtos da pesquisa e características sociais demográficas. Os dados foram analisados por estatística descritiva e regressão linear múltipla. Os resultados demonstraram que os estudantes universitários possuem CCEC moderado. Além disso, verificou-se que os construtos comportamento verde e motivadores externos exercem influência sobre o CCEC dos estudantes universitários, corroborando estudos anteriores, que utilizaram a mesma abordagem. Os resultados reforçam a necessidade de adoção de estratégias de marketing verde pelas empresas e maior envolvimento do poder público com aplicação de campanhas de conscientização ambiental, de modo que as gerações futuras possam desfrutar dos avanços econômicos em compatibilidade com a sustentabilidade ambiental.
\end{abstract}

Palavras-chave: Comportamento de consumo ecologicamente consciente. Marketing verde. Consumidor jovem. Motivadores externos. Comportamento verde.

Abstract: The present research aimed to identify the perception of the Ecologically conscious consumption behavior (CCEC) of university students through the readaptation of the research of Yeh (2015), whose work was inspired and adapted from the study of Zhao et al. (2014). The theoretical referential addresses the themes of green marketing, ecologically conscious consumption behavior, correlated studies about the structure of socially responsible consumer behavior among attitudes and behaviors favorable to the environment; and the systematization of behavioral variables, internal and external motivators that have an impact on the CCEC. A quantitative and descriptive research was carried out. Data were collected through the application of an online questionnaire directed to university students from the States of Bahia and Espírito Santo. The research instrument was composed of 32 statements structured with the research constructs and demographic social characteristics. Data were analyzed by descriptive statistics and multiple linear regression. The results showed that university students have moderate CCEC. In addition, it was found that the constructs green behavior and external motivators exert influence on the CCEC of university students, corroborating previous studies that used the same approach. The results reinforce the need to adopt green marketing strategies by companies and greater involvement of public Power through environmental awareness campaigns, so that future generations can enjoy the economic advances in compatibility with environmental sustainability

Keywords: Ecologically conscious consumption behavior. Green marketing. Young consumer. External motivators. Green Behavior.

\footnotetext{
${ }^{1}$ Mestre em Administração pela Fucape Business School. Analista Universitário da Uesb. E-mail: allenfigueiredo@uesb.edu.br.

${ }^{2}$ Mestrando em Administração pela Fucape Business School. Especialista em Gestão Estratégica pelo Centro Universitário São Camilo/ES. E-mail: maicontabelini@gmail.com.
}

Página 112 Caderno de Ciências Sociais Aplicadas, Vitória da Conquista/BA, vol. 14, n 24, ano 14, p. 112-132, jul/dez 2017. 


\section{Introdução}

Há anos vivencia-se a intervenção inadequada que as atividades industriais e dos mercados, que sustentam as sociedades capitalistas, exercem no meio ambiente. Os impactos provocados por essas atividades têm como consequências a extinção de matas e florestas, poluição, intempéries ambientais, entre outros, que afetam diretamente a qualidade de vida da população e comprometem a sobrevivência das gerações futuras e de toda a sociedade (PEREIRA PRADO et al., 2011).

Nesse contexto, as questões ambientais passaram a influenciar o comportamento dos consumidores, e consequentemente as estratégias de marketing das empresas. As empresas conceberam uma nova forma de obter lucros e alcançar uma posição de vantagem competitiva. Os consumidores comportam-se de forma diferenciada quando o assunto é decidir o que comprar. É necessário compreender todos os fatores determinantes para o comportamento socialmente consciente, visando o desenvolvimento adequado de estratégias de marketing (LAGES e NETO, 2002).

O termo marketing Verde foi discutido pela primeira vez em um seminário sobre "marketing ecológico", organizado pela American Marketing Association (AMA), em 1975, e tomou o seu lugar na literatura (BOZTEPE, 2012). O Marketing Verde ou Ambiental consiste em todas as atividades designadas para gerar e facilitar qualquer troca com o objetivo de satisfazer os desejos ou necessidades humanas, mediante esforços das organizações para que a satisfação das expectativas dos consumidores ocorra com o mínimo de impacto prejudicial sobre o meio ambiente ao passo que agrega valor para a empresa (LAROCHE et al., 1996; POLONSKY, 1994; ROBERTS, 1996).

Entendendo a relevância desse tema, este estudo propôs identificar o comportamento de consumo ecologicamente consciente (CCEC) de estudantes universitários, por meio da caracterização de variáveis e fatores internos e externos que exercem influência no CCEC, mediante a replicação parcial e readaptação da pesquisa de Yeh (2015), cujo trabalho foi inspirado e adaptado do estudo de Zhao et al. (2014). Realizou-se um estudo com abordagem quantitativa cujo público alvo foram os estudantes universitários de Instituições de Ensino Superior (pública e/ou privada) dos Estados da Bahia e Espírito Santo. Para coleta de dados foram utilizados de questionários estruturados.

O desenvolvimento deste trabalho buscou colaborar com a literatura do marketing ambiental por intermédio da compreensão dos fatores que influenciam o comportamento ecologicamente consciente dos consumidores jovens, buscando esclarecimentos para uma lacuna que permeia os estudos do CCEC: a lacuna entre a atitude favorável ao meio ambiente e o comportamento

Página 113 Caderno de Ciências Sociais Aplicadas, Vitória da Conquista/BA, vol. 14, n² 24, ano 14, p. 112-132, jul/dez 2017. 
ecologicamente consciente ou comportamento verde (YAM-TANG; CHAN, 1998; LAROCHE et al., 1996; LAROCHE et al., 2002; ZABKAR; HOSTA, 2013; ZHAO et al., 2014;). Uma melhor percepção dos fatores que influenciam o CCEC pode auxiliar no desenvolvimento de estratégias empresariais de marketing verde que gerem impactos positivos no CCEC (ZABKAR e HOSTA, 2013) e cooperar com políticas públicas de preservação ambiental e consumo ecologicamente consciente (CARRETE et al., 2012; LAROCHE et al., 2002).

O trabalho está estruturado em seis seções. Depois desta parte introdutória, a próxima seção apresenta a fundamentação teórica, onde foi revisada a literatura existente sobre comportamento de consumo ecologicamente consciente (CCEC), com ênfase nas variáveis que podem influenciar o CCEC. Na terceira seção, a metodologia da pesquisa é detalhada e as técnicas de análise de dados utilizados são descritas. Por fim, as considerações finais são apresentadas.

\section{Referencial teórico}

Um melhor entendimento dos atributos e do comportamento dos consumidores pró-ambientais tem sido pauta de muitos estudos na área de marketing ao longo dos anos (GONÇALVES-DIAS et al., 2009). Pesquisas sobre marketing verde tem procurado identificar o perfil de consumidores verdes usando diversas variáveis. Alguns estudiosos têm argumentado que o comportamento do consumidor verde é determinado por uma multiplicidade de fatores, dependendo do tipo de comportamento e envolvimento com o produto (CLEVELAND et al, 2005; ROBERTS; BACON, 1997). Não existe unanimidade sobre o meio mais efetivo de prever comportamentos de compra favoráveis ao meio ambiente (SCHLEGELMILCH et al., 1996).

Estudos a respeito do marketing ambiental têm empregado características sóciodemográficas, variáveis geográficas e parâmetros sociopsicográficos, sendo este último o que mais fornece subsídios para a definição de consciência social responsável (ANDERSON JR.; CUNNINGHAM,1972). O uso de indicadores comportamentais tem sido essencial para reduzir a potencial lacuna entre atitudes e comportamentos favoráveis ao meio ambiente (ROBERTS, 1996). Ademais, a identificação do consumidor verde tem se mostrado eficaz por meio da utilização de variáveis psicográficas ou comportamentais (STRAUGHAN e ROBERTS, 1999).

Não obstante, permanece a ausência de relação direta entre a consciência ecológica (atitude verde) e o efetivo comportamento de compra ou comportamento verde (YAM-TANG; CHAN, 1998;

Página 114 Caderno de Ciências Sociais Aplicadas, Vitória da Conquista/BA, vol. 14, n 24, ano 14, p. 112-132, jul/dez 2017. 
LAROCHE et al., 1996; LAROCHE et al., 2002; ZABKAR; HOSTA, 2013; ZHAO et al., 2014). A preocupação e consciência ambiental não garantem o comportamento de compra ecologicamente consciente (FOLLOWS e JOBBER, 2000). Nesse contexto, o entendimento dos comportamentos que motivam o consumo ambientalmente consciente é importante para o planejamento estratégico sustentável das empresas no intuito de oferecer bens e serviços direcionados para os indivíduos preocupados com as questões ambientais (LAGES e NETO, 2002).

Baseado na literatura do marketing ambiental e diante dos vários fatores que afetam o comportamento ecologicamente consciente do consumidor, a investigação desse trabalho foi conduzida por intermédio da replicação parcial (embasamento teórico e procedimentos metodológicos) e readaptação da pesquisa de Yeh (2015), cujo trabalho foi inspirado e adaptado do estudo de Zhao et al. (2014). A escolha pela extensão dos referidos estudos deveu-se à contemporaneidade das investigações e dos achados recentes, sobretudo, pela sistematização e nivelamento das variáveis (fatores comportamentais, motivadores internos e externos) que exercem impacto significativo sobre o CCEC, favorecendo a sua aplicação em outras conjunturas e diferentes universos de pesquisa.

Necessário sublinhar que a replicação de pesquisas se mostra como um caminho interessante para verificar as teorias (MORRISON et al., 2010). Permite verificar o mesmo fenômeno em outras situações, averiguando se os resultados prévios podem ser utilizados para outras populações ou são apenas características particulares (HUBBARD et al., 1998). Possibilitam também apurar resultados diferentes do estudo original, juntando os novos achados (MORRISON et al., 2010; TONCAR; MUNCH, 2010). Enfim, mesmo que a replicação não retorne os mesmos resultados do estudo antecessor, ela vai fornecer novos dados à pesquisa (HUNTER, 2001).

Isso posto, e baseado no estudo de Zhao et al. (2014), adaptado por Yeh (2015) os seguintes fatores (variáveis independentes) serão relacionados ao comportamento de consumo ecologicamente consciente (variável dependente): a) preocupação ambiental, b) eficácia percebida de consumo (EPC), c) atitude verde e d) comportamento verde; e e) estímulos governamentais, atuações das empresas e disponibilidade de produtos verdes.

\section{Preocupação ambiental}

A preocupação ambiental retrata a compreensão, para o indivíduo, que suas atitudes são importantes para a solução dos problemas ecológicos (AKEHURST et al., 2012). Os consumidores

Página 115 Caderno de Ciências Sociais Aplicadas, Vitória da Conquista/BA, vol. 14, n² 24, ano 14, p. 112-132, jul/dez 2017. 
conscientes são pessoas que buscar satisfazer suas necessidades individuais, e ao mesmo tempo demonstrar preocupação com o bem-estar social e ambiental (ANDERSON JR.; CUNNINGHAM,1972). Baseado nos aludidos argumentos, formulou-se a primeira hipótese da pesquisa: H1 - A Preocupação ambiental dos estudantes universitários é positivamente associada ao seu CCEC.

\section{Eficácia Percebida de Consumo (EPC)}

A Eficácia Percebida de Consumo (EPC), refere-se ao discernimento que os consumidores têm de que promovem ou não mudanças com suas atitudes e comportamento de consumo. A EPC demonstra o quanto o seu comportamento de consumo vai favorecer positivamente ou diminuir a agressão ao meio ambiente (GERSHOFF; FRELS, 2015). Se o consumidor não assimilar os efeitos positivos da sua ação pessoal, as possibilidades de contribuir para o ambiente reduzem radicalmente (TAN, 2011).

Identificar a percepção dos consumidores no sentido de que, ao realizar algum tipo de comportamento verde, pode colaborar com o meio ambiente, favorece o entendimento do CCEC (GERSHOFF; FRELS, 2015). Diante disso, elaborou-se a segunda hipótese do estudo: H2 - EPC dos estudantes universitários é positivamente associada ao seu CCEC.

\section{Atitude verde}

A atitude verde representa o desejo de se realizar um comportamento pró-ambiental. Os consumidores criam suas atitudes a partir de crenças e sentimentos, que podem ser relativamente positivos a marcas que estejam associadas a causas ambientais (PEREIRA, 2003). Entretanto, a intenção de compra do consumidor consciente ecologicamente é resultado de uma avaliação de tradeoff entre as consequências ambientais e as consequências individuais da compra (LAGES e NETO, 2002). As consequências individuais configuram-se como os motivos pelos quais alguns indivíduos com alta consciência ecológica não apresentam comportamento verde (FOLLOWS; JOBBER, 2000; LAGES e NETO, 2002). Denota-se a existência de uma hierarquia de fatores que precedem a compra dos produtos verdes: primeiro os valores do indivíduo, seguido das atitudes e das intenções, e, finalmente, a compra efetiva (FOLLOWS e JOBBER, 2000). Apoiado nesses pressupostos,

Página 116 Caderno de Ciências Sociais Aplicadas, Vitória da Conquista/BA, vol. 14, nº 24, ano 14, p. 112-132, jul/dez 2017. 
descrevemos a terceira hipótese da investigação: H3 - A Atitude verde dos estudantes universitários é positivamente associada ao seu CCEC.

\section{Comportamento verde}

A utilização de indicadores comportamentais tem sido essencial para reduzir a potencial lacuna entre atitudes e comportamentos favoráveis ao meio ambiente (ROBERTS, 1996). O comportamento verde é classificado em três tipos: uso/reuso, compra e reciclagem (ZHAO et al., 2014). Em relação ao comportamento verde de compra, tem sido observado um obstáculo entre a realidade e a percepção dos consumidores sobre os benefícios dos produtos verdes (TSENG e HUNG, 2013). Diante de tal relevância, formulou-se a quarta hipótese: H4 - O Comportamento verde dos estudantes universitários é positivamente associado ao seu CCEC.

\section{Motivadores externos}

Motivadores externos podem ser entendidos como elementos extrínsecos ao indivíduo que podem favorecer ou descomplicar a realização de CCEC, por exemplo, políticas públicas ou incentivos governamentais, campanhas empresariais de produtos verdes e a facilidade de encontrar produtos ou serviços verdes (LAGES e NETO, 2002). A disponibilidade de produtos verdes também é um fator externo, cuja falta favorece o aumento da lacuna entre uma atitude verde e a realização do respectivo comportamento verde. Considerando que os motivadores externos podem exercer influência sobre o CCEC, desenvolveu-se a quinta hipótese do estudo: H5 - Motivadores externos dos estudantes universitários são positivamente associados ao seu CCEC.

\section{Modelo proposto}

Resultante desta revisão de literatura e evidenciada as hipóteses de pesquisa, concebeu-se o seguinte modelo conceitual, que sistematiza a relação das referidas variáveis com o CCEC:

Página 117 Caderno de Ciências Sociais Aplicadas, Vitória da Conquista/BA, vol. 14, n 24, ano 14, p. 112-132, jul/dez 2017. 
Figura 1: Modelo proposto de possíveis fatores que afetam o CCEC, readaptado de Yeh (2015).

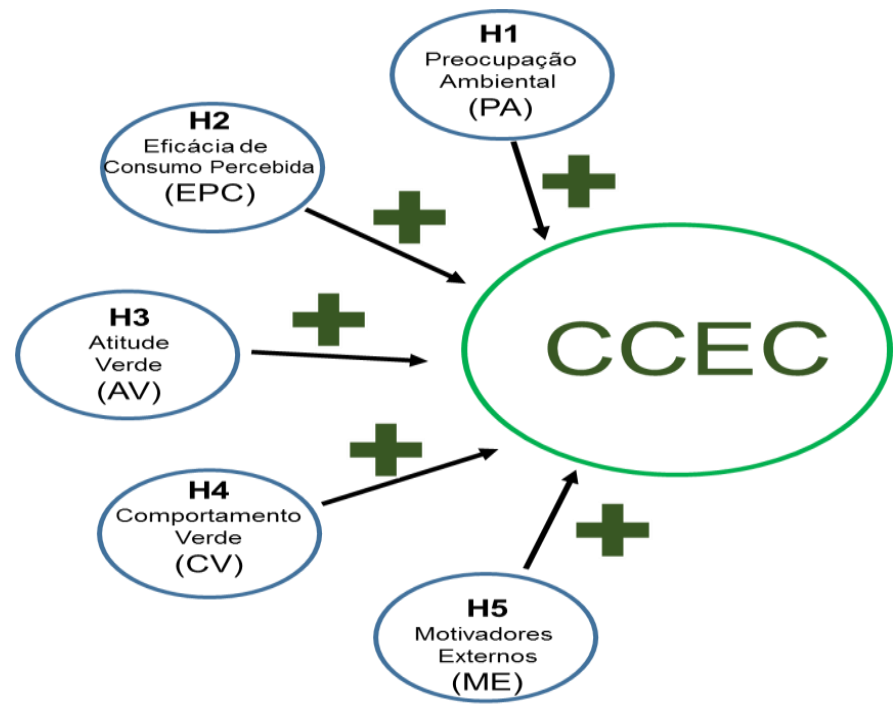

Fonte: Elaboração própria.

\section{Metodologia}

A pesquisa teve como objetivo geral investigar as variáveis que exercem influência no comportamento de compra ecologicamente consciente (variável dependente). As variáveis independentes correlacionadas com o CCEC foram: a) preocupação ambiental, b) eficácia percebida de consumo (EPC), c) atitude verde, d) comportamento verde; e e) motivadores externos (estímulos governamentais, atuações das empresas e disponibilidade de produtos verdes). A metodologia utilizada foi a pesquisa quantitativa e descritiva, com coleta de dados por meio de reaplicação do questionário desenvolvido por Yeh (2015), adaptado do estudo de Zhao et al. (2014).

A amostra é não probabilística de tipo por conveniência. A escolha pela população alvo (estudantes universitários) deveu-se à importância de mensurar o futuro do consumo consciente (STRAUGHAN e ROBERTS, 1999) e, levando em consideração que os mesmos têm à sua disposição uma quantidade crescente de informações ambientais (CÔRTES e DO AMARAL MORETTI, 2013), fazem parte de um público consumidor crítico e formador de opinião e representam um grupo social que pode ser considerado como um dos principais agentes para transformar a forma de consumir produtos e serviços (GROHMANN et al., 2012) propiciando às gerações futuras avanços econômicos em compatibilidade com a sustentabilidade ambiental. A amostra foi constituída de 426 respondentes.

Página 118 Caderno de Ciências Sociais Aplicadas, Vitória da Conquista/BA, vol. 14, n 24, ano 14, p. 112-132, jul/dez 2017. 
No desenvolvimento deste estudo foi utilizado o levantamento de dados (survey) como técnica de coleta, por intermédio de disponibilização de formulário online e contato via e-mail com os respondentes das instituições de ensino superior (IES) nos meses de outubro e novembro de 2015. O questionário elaborado com perguntas fechadas de auto resposta com escala de avaliação de 5 pontos, tipo Likert, variando de "discordo totalmente" até "concordo totalmente" foi baseado em escalas já validadas por trabalhos anteriores.

O instrumento de pesquisa, consistiu de três partes. A primeira seção compreendia 20 afirmativas que buscavam observar os seguintes construtos: eficácia percebida de consumo, atitudes em relação ao consumo verde, a preocupação ambiental, motivadores externos e o comportamento do consumidor verde. A segunda parte solicitava a autoavaliação do respondente sobre seu próprio CCEC (variável dependente deste estudo). Em seguida, o questionário também coletou informações demográficas, incluindo idade, gênero e renda.

O quadro 1 sintetiza a abordagem dos construtos nas questões do instrumento de pesquisa, auxiliando na compreensão e apresentação dos mesmos, cujas siglas também serão utilizadas na seção de análise dos dados. Nesse sentido, tem-se o seguinte:

Quadro 1: Questionário readaptado do instrumento de pesquisa de Yeh (2015)

\begin{tabular}{|c|c|}
\hline \multirow{2}{*}{\multicolumn{2}{|c|}{ SESSÃO I }} \\
\hline & \\
\hline \multicolumn{2}{|c|}{ Eficácia Percebida de Consumo (EPC) } \\
\hline & Eu não posso fazer nada para ajudar no controle da poluição do meio ambiente. (R) \\
\hline 3. & Meu comportamento pode ter efeito positivo ao meio ambiente ao comprar produtos verdes ou ecológicos. \\
\hline \multicolumn{2}{|r|}{ Atitude Verde (AV) } \\
\hline 4. & É mais conveniente comprar aparelhos do que repará-los. (R) \\
\hline $\begin{array}{l}5 . \\
(\mathrm{R})\end{array}$ & Os recursos consumidos por mim mesmo são poucos e não causam qualquer poluição para o meio ambiente. \\
\hline 6. & Não é preciso persuadir os outros para se envolverem com comportamento verde. (R) \\
\hline & É muito importante centralizar esforços na economia de água e energia de uso doméstico. \\
\hline \multicolumn{2}{|r|}{$\begin{array}{l}\text { 8. É muito importante, no cenário atual, um rigoroso direcionamento rumo ao comportamento verde (ou } \\
\text { ecológico). }\end{array}$} \\
\hline & Eu apoio o sistema de "pagar para usar sacolas plásticas". \\
\hline \multicolumn{2}{|c|}{ Preocupação Ambiental (PA) } \\
\hline 10. & O equilíbrio da natureza é muito delicado e fácil de ser perturbado. \\
\hline 11. & A humanidade está abusando severamente do meio ambiente. \\
\hline 12. & Todas as questões pertinentes à poluição me deixam desconfortáveis. \\
\hline \multicolumn{2}{|c|}{ Moderadores Externos (ME) } \\
\hline 13. & As campanhas publicitárias de produtos verdes têm efeito sobre minhas compras. \\
\hline 14. & As campanhas do governo me induzem a me preocupar com o meio ambiente. \\
\hline & Eu acho facilmente produtos verdes para comprar. \\
\hline \multicolumn{2}{|c|}{ Comportamento Verde (CV) } \\
\hline & Eu, sempre que possível, uso produtos descartáveis. \\
\hline
\end{tabular}

Página 119 Caderno de Ciências Sociais Aplicadas, Vitória da Conquista/BA, vol. 14, nº 24, ano 14, p. 112-132, jul/dez 2017. 


\begin{tabular}{|ll|}
\hline 17. & Eu tento comprar lâmpadas com melhor eficiência para poupar energia. \\
\hline 18. & Eu tento comprar aparelhos com eficiência em consumo de energia. \\
\hline 19. & Eu, sempre que possível, reuso sacolas plásticas ou de papelão. \\
\hline 20. & Eu, sempre que possível, reuso água. \\
\hline 21. & Eu, sempre que possível, vendo/destino garrafas plásticas para o centro de reciclagem. \\
\hline SESSÃO II \\
\hline Comportamento de Consumo Ecologicamente Consciente (CCEC) \\
\hline 22. & Eu me considero um consumidor ecologicamente consciente. (R) \\
\hline 23. & Eu tenho comportamento de consumo responsável com o meio ambiente. \\
\hline 24. & Os meus hábitos de consumo são determinados, prioritariamente, por razões ecológicas. \\
\hline 25. & Minhas atitudes de compra são favoráveis ao consumo de produtos verdes. \\
\hline 26. & Quando eu posso escolher, prefiro comprar produtos que causem menor prejuízo ambiental, independente do \\
\hline preço. & \\
\hline SESSÃO III \\
\hline Questões demográficas \\
\hline 27. & Qual o seu gênero? \\
\hline 28. & Qual é a sua idade em anos (responder apenas com números)? \\
\hline 29. & A sua Instituição de Ensino Superior (IES) é: \\
\hline 30. & Qual o Estado que você mora? \\
\hline 31. & Você trabalha? \\
\hline 32. & Qual sua renda familiar? \\
\hline
\end{tabular}

As perguntas invertidas (reversed) estão identificadas com (R)

Fonte: Yeh (2015)

As afirmativas das questões: 2 (EPC); 5, 6 e 7 (AV); e 22 (CV), foram invertidas no questionário, visando não comprometer a média dos respectivos construtos na análise dos dados. Considerando que a "concordância" com a afirmativa das referidas questões seriam respostas favoráveis ao CCEC, ao inverter as afirmativas ocorre o contrário, ou seja, a resposta favorável será a “discordância". Tal procedimento foi necessário, para que houvesse coerência na média dos construtos.

\section{Análise dos dados}

Inicialmente, realizou-se uma análise demográfica da amostra do estudo e das estatísticas descritiva das variáveis. Em seguida é apresentada a análise da regressão múltipla das médias do construto CCEC (variável dependente) com as médias das questões que formam os construtos utilizados como variáveis independentes. A análise dos dados coletados foi realizada com auxílio de software estatístico.

Conforme demonstrado na tabela 1, a ampla maioria dos respondentes (90,6\%) possui idade compreendida entre 17 e 29 anos. Os demais participantes estão na faixa etária adulta (30 a 59 anos). $\mathrm{Na}$ concepção deste estudo partiu-se da ideia de que os jovens estão crescendo e vivendo em uma 
época de degradação ambiental e, portanto, estariam mais predispostos a defender e adotar aas questões ecológicas (STRAUGHAN e ROBERTS, 1999).

Tabela 1: Perfil da amostra ( $\mathrm{n}=426)$

\section{Gênero}

Feminino

\section{Idade}

\section{Instituição de Ensino Superior}

Pública

\section{Estado}

Espírito Santo

Bahia

Outros

\section{Trabalha?}

Não

Sim

\section{Renda Familiar} (em salário mínimo)

Até 1,5

1,5 a 3

3 a 8

8 a15

Mais de 15

$\begin{array}{cc}147 & 34,5 \\ 276 & 64,8 \\ 3 & , 7 \\ & \\ 205 & 48.1 \\ 221 & 51.9\end{array}$

$186-43.7$

$142 \quad 33.3$

$\begin{array}{ll}77 & 18.1\end{array}$

$18 \quad 4.2$

3

Fonte: Dados da pesquisa

No tocante à renda familiar, a maior parte dos respondentes informou que estão inseridos na faixa de renda de até 1,5 salário mínimo (43,7\%), seguidos por estudantes de renda familiar compreendida entre 1,5 a 3 salários mínimos (33,3\%). Percebe-se que a grande maioria da amostra possui baixa ou média renda familiar. As pessoas com níveis de renda mais alto estão mais inclinados a arcar com os custos agregados aos produtos verdes, e dessa forma, a renda pode exercer impacto 
positivo no comportamento verde (ANDERSON JR.; CUNNINGHAM,1972; ROBERTS, 1996; ROBERTS; BACON, 1997; STRAUGHAN; ROBERTS, 1999).

Quase dois terços dos participantes eram estudantes de universidades públicas (61,7\%). Além disso, prevaleceu os respondentes que residem no estado da Bahia (64,8\%). Esses dados são reflexo do acesso aos respondentes de acordo a conveniência dos pesquisadores.

\section{Estatísticas descritivas da amostra}

As questões (itens das escalas) do instrumento de pesquisa foram agrupadas em construtos para facilitar o cálculo e a interpretação da média e favorecer a compreensão de quais construtos estão efetivamente associados ao CCEC, conforme tabela 2.

Tabela 2: Estatística descritiva da amostra de estudantes universitários

\begin{tabular}{|c|c|c|c|c|}
\hline Código & Afirmativas & $\mathbf{n}$ & Média & $\begin{array}{l}\text { Desvio } \\
\text { Padrão }\end{array}$ \\
\hline E1 & $\begin{array}{l}\text { Eu não posso fazer nada para ajudar no controle da poluição do meio } \\
\text { ambiente. }(\mathrm{R})\end{array}$ & 426 & 4.4155 & 1.16766 \\
\hline E2 & $\begin{array}{l}\text { Meu comportamento pode ter efeito positivo ao meio ambiente ao comprar } \\
\text { produtos verdes ou ecológicos. }\end{array}$ & 426 & 3.9836 & 1.26014 \\
\hline EPC & Construto Eficácia Percebida de Consumo & 426 & 4.1995 & .95430 \\
\hline A1 & É mais conveniente comprar aparelhos do que repará-los. (R) & 426 & 3.5235 & 1.20222 \\
\hline A2 & $\begin{array}{l}\text { Os recursos consumidos por mim mesmo são poucos e não causam } \\
\text { qualquer poluição para o meio ambiente. (R) }\end{array}$ & 426 & 3.8216 & 1.17867 \\
\hline A3 & $\begin{array}{l}\text { Não é preciso persuadir os outros para se envolverem com comportamento } \\
\text { verde. }(\mathrm{R})\end{array}$ & 426 & 3.9202 & 1.28821 \\
\hline A4 & $\begin{array}{l}\text { É muito importante centralizar esforços na economia de água e energia de } \\
\text { uso doméstico. }\end{array}$ & 426 & 4.3427 & 1.19800 \\
\hline A5 & $\begin{array}{l}\text { É muito importante, no cenário atual, um rigoroso direcionamento rumo ao } \\
\text { comportamento verde (ou ecológico). }\end{array}$ & 426 & 4.3169 & 1.17044 \\
\hline A6 & Eu apoio o sistema de “pagar para usar sacolas plásticas”. & 426 & 3.3122 & 1.45641 \\
\hline $\mathbf{A V}$ & Construto Atitude Verde & 426 & 3.8728 & .67626 \\
\hline $\mathrm{P} 1$ & O equilíbrio da natureza é muito delicado e fácil de ser perturbado. & 426 & 3.8850 & 1.29375 \\
\hline $\mathrm{P} 2$ & A humanidade está abusando severamente do meio ambiente. & 426 & 4.4859 & 1.02947 \\
\hline P3 & Todas as questões pertinentes à poluição me deixam desconfortáveis. & 426 & 3.3498 & 1.36931 \\
\hline PA & Construto Preocupação Ambiental & 426 & 3.9069 & .88321 \\
\hline M1 & $\begin{array}{l}\text { As campanhas publicitárias de produtos verdes têm efeito sobre minhas } \\
\text { compras. }\end{array}$ & 426 & 3.1948 & 1.05700 \\
\hline M2 & $\begin{array}{l}\text { As campanhas do governo me induzem a me preocupar com o meio } \\
\text { ambiente. }\end{array}$ & 426 & 2.9202 & 1.30094 \\
\hline M3 & Eu acho facilmente produtos verdes para comprar. & 426 & 2.4225 & 1.19994 \\
\hline ME & Construto Motivadores Externos & 426 & 2.8459 & .84790 \\
\hline $\mathrm{C} 1$ & Eu, sempre que possível, uso produtos descartáveis. & 426 & 3.2300 & 1.22662 \\
\hline $\mathrm{C} 2$ & Eu tento comprar lâmpadas com melhor eficiência para poupar energia. & 426 & 4.2207 & 1.19927 \\
\hline C3 & Eu tento comprar aparelhos com eficiência em consumo de energia. & 426 & 4.0376 & 1.23231 \\
\hline
\end{tabular}

Página 122 Caderno de Ciências Sociais Aplicadas, Vitória da Conquista/BA, vol. 14, n 24, ano 14, p. 112-132, jul/dez 2017. 


\section{- Cadernos de Ciénclas SOCIAIS APLICADAS}

\begin{tabular}{c|l|c|c|c}
\hline C4 & Eu, sempre que possível, reuso sacolas plásticas ou de papelão. & 426 & 4.1080 & 1.30071 \\
\hline C5 & Eu, sempre que possível, reuso água. & 426 & 3.5070 & 1.40292 \\
\hline C6 & $\begin{array}{l}\text { Eu, sempre que possível, vendo/destino garrafas plásticas para o centro de } \\
\text { reciclagem. }\end{array}$ & 426 & 2.7840 & 1.44396 \\
\hline $\mathbf{C V}$ & Construto Comportamento Verde & $\mathbf{4 2 6}$ & $\mathbf{3 . 6 4 7 9}$ & $\mathbf{. 7 1 5 2 2}$ \\
\hline CCEC1 & Eu me considero um consumidor ecologicamente consciente. (R) & 426 & 3.1784 & .96338 \\
\hline CCEC2 & Eu tenho comportamento de consumo responsável com o meio ambiente. \\
\hline CCEC3 & $\begin{array}{l}\text { Os meus hábitos de consumo são determinados, prioritariamente, por } \\
\text { razões ecológicas. }\end{array}$ & 426 & 3.3498 & .98803 \\
\hline CCEC4 & Minhas atitudes de compra são favoráveis ao consumo de produtos verdes. & 426 & 3.0798 & 1.04831 \\
\hline CCEC5 & $\begin{array}{l}\text { Quando eu posso escolher, prefiro comprar produtos que causem menor } \\
\text { prejuízo ambiental, independente do preço. }\end{array}$ & 426 & 3.0704 & 1.25734 \\
\hline CCEC & Comportamento de Consumo Ecologicamente Consciente & $\mathbf{4 2 6}$ & $\mathbf{3 . 1 1 6 4}$ & $\mathbf{. 8 1 5 0 1}$ \\
\hline
\end{tabular}

Fonte: Dados da pesquisa

Analisando as estatísticas descritivas dos construtos abordados no estudo verificou-se que a eficácia percebida de consumo (EPC) apresentou a maior média $(M=4.1995)$. A EPC é considerada em muitos estudos anteriores como um dos elementos de maior impacto no comportamento do consumidor ecologicamente consciente (ROBERTS, 1996; STRAUGHAN e ROBERTS, 1999). A afirmativa invertida "Eu não posso fazer nada para ajudar no controle da poluição do meio ambiente" $(M=4,41)$ alcançou a segunda maior média de todas as afirmativas, de modo que pode-se deduzir que os estudantes universitários compreendem que o seu comportamento ecologicamente consciente pode ser benéfico para o meio ambiente.

A segunda maior média foi demonstrada pelo construto preocupação ambiental $(M=3.9069)$, que em muitos estudos é concebida como um fator que impacta positivamente no CCEC (PAÇO e RAPOSO, 2010; ROBERTS e BACON, 1997). Importante destacar que a afirmativa "A humanidade está abusando severamente do meio ambiente" $(M=4.4859)$, pertencente a este construto, apresentou a maior média de todas as afirmativas do instrumento de pesquisa, comprovando que os estudantes universitários têm conhecimento e estão preocupados com as questões ecológicas. Embora tenham apresentado as maiores médias entre os construtos, a eficácia percebida de consumo $(\mathrm{EPC}, \mathrm{DP}=0,95)$ seguida do construto preocupação ambiental ( $\mathrm{PA}, \mathrm{DP}=0,88)$ também demonstraram menor concordância entre as respostas do questionário.

Por outro lado, o construto que apresentou menor média foi Motivadores Externos $(\mathrm{M}=2.8459)$, com destaque para a afirmativa com menor média: "Eu acho facilmente produtos verdes para comprar" $(M=2,42)$, evidenciando a indisponibilidade ou insuficiência de produtos ecológicos no mercado. Além disso, as afirmativas inerentes às campanhas publicitárias e políticas governamentais

Página 123 Caderno de Ciências Sociais Aplicadas, Vitória da Conquista/BA, vol. 14, nº 24, ano 14, p. 112-132, jul/dez 2017. 
tiveram médias moderadas baixas, deixando claro a inexistência de políticas públicas para conscientização dos indivíduos e principalmente da ausência de estratégias de marketing verde pelas empresas, buscando fomentar e direcionar os produtos ecológicos para o seu público alvo, configurando-se em uma falha para atingir o comportamento verde.

Os construtos atitude verde $(\mathrm{CV}, \mathrm{DP}=0,67)$ e comportamento verde (CV, $\mathrm{DP}=0,71)$ apresentaram maior concordância nas respostas dos questionários. A atitude verde precede o comportamento verde e representa a vontade do indivíduo em exercer um comportamento próambiental. Analisando individualmente as escalas do construto atitude verde, a afirmativa "É muito importante centralizar esforços na economia de água e energia de uso doméstico” ( $M=4.34)$, obteve a maior média. Esta afirmativa demonstra que o indivíduo está mais propenso a adotar atitudes verdes que primeiramente lhe traga um benefício individual (economia financeira na redução das contas de água e energia), deixando em segundo planos outras atitudes favoráveis ao meio ambiente, a exemplo da afirmativa "Eu apoio o sistema de pagar para usar sacolas plásticas" (M=3.31).

O construto comportamento verde, teve situação parecida, ou seja, o indivíduo demonstra uma hierarquia de valores, onde prevalecem o peso das consequências individuais, pois demonstra alta predisposição para o comportamento verde quando enxerga algum benefício particular, a exemplo das afirmativas: "Eu tento comprar lâmpadas com melhor eficiência para poupar energia" (M=4.2207) e "Eu tento comprar aparelhos com eficiência em consumo de energia $(M=4.0376)$, que lhe proporcionará economia financeira e concomitantemente beneficiará o meio ambiente. Entretanto não demonstra essa mesma disposição para destinar produtos para reciclagem, como comprova a afirmativa "Eu, sempre que possível, vendo/destino garrafas plásticas para o centro de reciclagem" $(M=2.7840)$, que apresentou a segunda menor média entre as afirmativas.

Examinando a autoavaliação dos indivíduos da amostra é possível inferir que os estudantes universitários demonstram ter CCEC moderado $(\mathrm{M}=3,11)$. Umas dessas afirmativas: "Os meus hábitos de consumo são determinados, prioritariamente, por razões ecológicas” $(M=2,9)$, elaborada com o intuito de avaliar, de forma direta, o nível de prioridade que as questões ecológicas ocupam no CCEC dos estudantes universitários, foi a que obteve menor média nesse construto.

Página 124 Caderno de Ciências Sociais Aplicadas, Vitória da Conquista/BA, vol. 14, n 24, ano 14, p. 112-132, jul/dez 2017. 


\section{Análise da regressão linear múltipla}

Os resultados obtidos no cálculo da regressão linear múltipla (tabela 3) demonstram que o modelo final encontrado explica $25,7 \%\left(\mathrm{R}^{2}\right.$ ajustado $\left.=0,257\right)$ da relação dos construtos apontados como significativos com o CCEC. Trata-se de um percentual razoável levando-se em consideração os vários fatores que podem impactar no comportamento de consumo ecológico e a dificuldade de reunir tantas variáveis independentes em um único modelo.

Tabela 3: Modelo da regressão linear múltipla - variável dependente CCEC de estudantes universitários

\begin{tabular}{|c|c|c|c|c|c|c|c|c|c|c|}
\hline \multirow{3}{*}{$\begin{array}{c}\text { Modelo } \\
2\end{array}$} & \multirow[b]{2}{*}{$\mathrm{R}$} & \multirow[b]{2}{*}{$\begin{array}{c}\mathrm{R} \\
\text { quadrado }\end{array}$} & \multirow[b]{2}{*}{$\begin{array}{c}\mathrm{R} \\
\text { quadrado } \\
\text { ajustado }\end{array}$} & \multirow[b]{2}{*}{$\begin{array}{c}\text { Erro } \\
\text { padrão da } \\
\text { estimativa }\end{array}$} & \multicolumn{5}{|c|}{ Estatísticas de mudança } & \multirow[b]{2}{*}{$\begin{array}{l}\text { Durbin- } \\
\text { Watson }\end{array}$} \\
\hline & & & & & $\begin{array}{c}\text { Alteração } \\
\text { de } \mathrm{R} \\
\text { quadrado }\end{array}$ & $\begin{array}{c}\text { Alteração } \\
\text { F }\end{array}$ & df1 & df2 & $\begin{array}{c}\text { Sig. } \\
\text { Alteração } \\
\text { F }\end{array}$ & \\
\hline & ,511b & .261 & .257 & .70232 & .075 & 43.167 & 1 & 423 & .000 & 1.489 \\
\hline
\end{tabular}

b. Preditores: (Constante), CV, ME

c. Variável Dependente: CCEC

Método de estimação: Stepwise

Testes de validez:

ANOVA: significativo

Teste de Aleatoriedade: Suporta a hipótese de Aleatoriedade

Teste de Aderência Kolmogorov-Smirnov: Suporta hipótese de Aderência a distribuição normal

Teste de Homocedasticidade: Suporta a hipótese de homocedasticidade

Fonte: Dados da pesquisa

Confrontando os resultados da regressão realizada com as hipóteses do estudo, encontrou-se os seguintes resultados:

1. Preocupação ambiental dos estudantes universitários é positivamente associada ao seu CCEC. Foi rejeitada.

H2. EPC dos estudantes universitários é positivamente associada ao seu CCEC. Foi rejeitada.

H3. Atitude verde dos estudantes universitários é positivamente associada ao seu CCEC. Foi rejeitada.

H4. Comportamento verde dos estudantes universitários é positivamente associado ao seu CCEC. Foi suportada.

Página 125 Caderno de Ciências Sociais Aplicadas, Vitória da Conquista/BA, vol. 14, n 24, ano 14, p. 112-132, jul/dez 2017. 


\section{- Cadernos de Ciénclas SOCIAIS APLICADAS}

H5. Motivadores externos dos estudantes universitários são positivamente associados ao seu CCEC. Foi suportada.

O modelo evidenciado na tabela 4 demonstra ainda que, para a amostra estudada, somente as variáveis comportamento verde e motivadores externos foram estatisticamente significativas $(p<0,05)$ e, portanto, exercem poder preditivo sobre o comportamento de consumo ecologicamente consciente (CCEC) de estudantes universitários.

Os indicadores comportamentais têm sido os mais indicados para predizerem o consumo ecologicamente consciente (ROBERTS, 1996). Além da atividade da compra propriamente, o comportamento verde engloba também as atividades de uso/reuso e reciclagem, proporcionando ao indivíduo diversas formas de manifestar seu comportamento pró-ambiental.

Os motivadores externos colaboram para a consciência ecológica e exercem influência no comportamento ecologicamente consciente, trata-se de estímulos vindos de campanhas empresariais e governamentais, facilidade de acesso aos produtos verde e a disponibilidade dos mesmos aos indivíduos.

Em estudos anteriores (ZHAO et al., 2014; YEH, 2015) o comportamento verde e os motivadores externos foram fatores que demonstraram poder de predição positiva no comportamento ambientalmente consciente.

Tabela 4: coeficientes estimados da variável dependente CCEC de estudantes universitários

\begin{tabular}{|c|c|c|c|c|c|c|c|c|c|c|c|c|}
\hline \multirow{2}{*}{$\begin{array}{c}\text { Modelo } \\
2\end{array}$} & \multicolumn{2}{|c|}{$\begin{array}{c}\text { Coeficientes não } \\
\text { padronizados }\end{array}$} & \multirow{2}{*}{$\begin{array}{c}\text { Coeficientes } \\
\text { padronizados } \\
\text { Beta }\end{array}$} & \multirow[b]{2}{*}{$t$} & \multirow[b]{2}{*}{ Sig. } & \multicolumn{2}{|c|}{$\begin{array}{c}95,0 \% \\
\text { Intervalo de } \\
\text { Confiança } \\
\text { para B }\end{array}$} & \multicolumn{3}{|c|}{ Correlações } & \multicolumn{2}{|c|}{$\begin{array}{l}\text { Estatísticas de } \\
\text { colinearidade }\end{array}$} \\
\hline & B & $\begin{array}{l}\text { Erro } \\
\text { Padrão }\end{array}$ & & & & 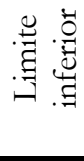 & 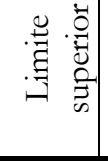 & 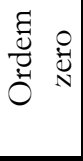 & .⿱乛తు: & ت્屯 & 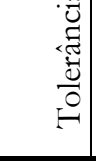 & 声 \\
\hline $\begin{array}{l}\text { (Const } \\
\text { ante) }\end{array}$ & .764 & .197 & & 3.888 & .000 & .378 & 1.151 & & & & & \\
\hline $\mathrm{CV}$ & .436 & .048 & .382 & 9.008 & .000 & .341 & .531 & .431 & .401 & .377 & .970 & 1.031 \\
\hline $\mathrm{ME}$ & .268 & .041 & .279 & 6.570 & .000 & .188 & .348 & .345 & .304 & .275 & .970 & 1.031 \\
\hline
\end{tabular}

a. Variável Dependente: CCEC

Fonte: Dados da pesquisa

Página 126 Caderno de Ciências Sociais Aplicadas, Vitória da Conquista/BA, vol. 14, n 24, ano 14, p. 112-132, jul/dez 2017. 
Embora tenha alcançado a maior média entre os construtos estudados, a eficácia percebida de consumo que é considerada em muitos estudos anteriores como um dos elementos de maior impacto no comportamento do consumidor ecologicamente consciente (ROBERTS, 1996; STRAUGHAN e ROBERTS, 1999), não se foi estatisticamente significativa na relação com o CCEC de estudantes universitários.

Da mesma forma, a preocupação ambiental, apesar de ter apresentado a segunda maior média entre os construtos estudados, não exibiu significância estatística ao ser relacionada com o CCEC de estudantes universitários. Uma possível explicação está no fato de que embora os indivíduos demonstrem estar preocupados com o meio ambiente, grande parte não exerce atitude verde. Pode ser que acreditem que a preservação ambiental é dever do poder público e das empresas que consomem os recursos naturais, ou ainda não estão dispostos a arcar com os custos decorrentes da preservação ecológica que são incrementados nos preços dos produtos verdes, de modo que a preocupação ambiental não conduz ao comportamento ecologicamente correto (MAIBACH, 1993).

O outro construto que não demonstrou poder preditivo sobre o CCEC de estudantes universitários foi a atitude verde. Conforme sublinhado na literatura desta pesquisa, persiste a lacuna entre a atitude pró-ambiental e sua transformação efetiva em comportamento verde (YAM-TANG; CHAN, 1998; LAROCHE et al., 1996; LAROCHE et al., 2002; ZABKAR; HOSTA, 2013; ZHAO et al., 2014).

A figura 2 ilustra o modelo final do estudo:

Página 127 Caderno de Ciências Sociais Aplicadas, Vitória da Conquista/BA, vol. 14, nº 24, ano 14, p. 112-132, jul/dez 2017. 
Figura 2: Construtos significativos para o CCEC dos estudantes universitários pelo resultado da regressão.

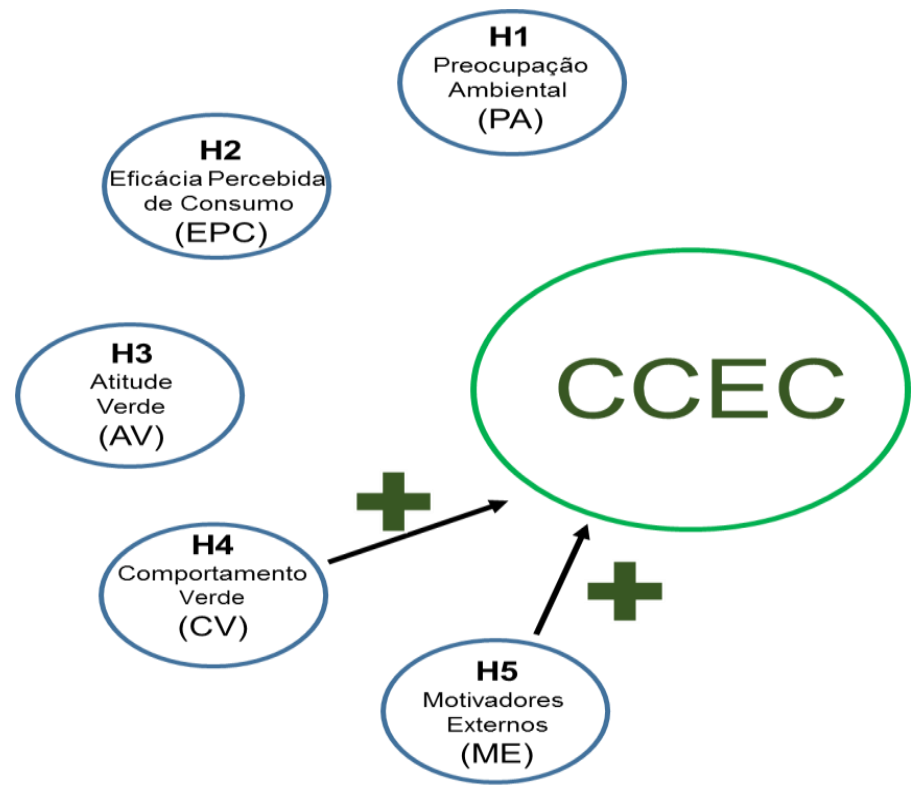

Fonte: Elaboração própria

\section{Considerações finais}

A apreensão da coletividade em relação às questões ambientais motivou o desenvolvimento de vários estudos com o objetivo de compreender o comportamento do consumidor. Este trabalho buscou colaborar com a literatura buscando identificar, de forma específica, a percepção do CCEC dos estudantes universitários em relação a construtos utilizados em estudos recentes (ZHAO et al., 2014; YEH, 2015).

No campo prático, os estudos apontam para a necessidade do desenvolvimento de estratégias, com a utilização do Marketing Verde, objetivando conhecer e diferenciar o consumidor ecologicamente consciente e visando ao desenvolvimento de campanhas de estímulo da consciência ambiental e aumentar a oferta de produtos verdes direcionada para o público que demonstrem maior predisposição para aquisição de produtos verdes. A amostra estudada neste artigo é de uma população relativamente jovem e que é influenciada pelos fatores externos e pelo comportamento verde, conforme suportado nas hipóteses do estudo. A formação de indivíduos pensantes no ecologicamente correto é de suma importância para o futuro do meio ambiente, principalmente no que se refere a criação de ideias sustentáveis dentro das organizações, como embalagens ecológicas, processos de logística reversa e reaproveitamento de materiais, seja por meio de insumos processados ou o usufruto de uma matéria-

Página 128 Caderno de Ciências Sociais Aplicadas, Vitória da Conquista/BA, vol. 14, n 24, ano 14, p. 112-132, jul/dez 2017. 
prima resultante de um processo de reciclagem correto. O poder público também deve se inserir no processo de consumo responsável, principalmente por meio de ações de políticas voltadas à conscientização ecológica.

Dentre as limitações, ressalta-se que a amostra de estudantes foi coletada por conveniência dos pesquisadores. Contudo, a investigação alcançou sua relevância e teve o seu objetivo respondido, apesar desta limitação. O questionário estruturado também limita outras possíveis linhas de pensamento, ou seja, o entrevistado não tem espaço para expressar ideias e opiniões mais aprofundadas e detalhadas.

Em comparação com o estudo original (YEH, 2015) este trabalho apresentou homogeneidade. O modelo final encontrado para CCEC de estudantes universitários apontou como significativas as variáveis comportamento verde (CV) e motivadores externos (ME) que juntas explicam 25,7\% da relação dos construtos apontados como significativos com o CCEC. O modelo do CCEC de Yeh (2015), utilizando amostra da população brasileira, obteve significância nas variáveis comportamento verde (CV), motivadores externos (ME) e eficácia percebida de consumo (EPC) que explicaram 26,5\% da relação com o CCEC.

Por fim, sugere-se que em outras possíveis pesquisas futuras, proceda-se uma maior ampliação geográfica do universo da amostra, cujo instrumento de pesquisa fique à disposição dos potenciais respondentes por um tempo mais amplo. Também poderá ser testado um estudo com entrevistas semiestruturadas, onde é possível uma abertura de opinião, tornando a pesquisa em um diálogo que pode levar a várias sugestões e diretrizes ainda não exploradas e que podem conduzir ao alcance de outros resultados. Deve-se também avaliar a incorporação de mais variáveis no modelo, de modo a alcançar maior poder explicativo, e colaborar decisivamente com o melhor entendimento do CCEC, pois, mesmo diante de tantos estudos realizados, permanece a noção que conforme se aumenta a preocupação ambiental das pessoas, simultaneamente também aumenta a complexidade e os possíveis fatores que podem influenciar o CCEC dos indivíduos.

\section{Referências}

AKEHURST, Gary; AFONSO, Carolina; MARTINS GONÇALVES, Helena. Re-examining green purchase behaviour and the green consumer profile: New evidences. Management Decision, v. 50, $\mathrm{n}$. 5, p. 972-988, 2012.

ANDERSON JR, W. Thomas; CUNNINGHAM, Willian H. "The Socially Conscious Consumer". Journal of Marketing, v. 36, July, 1972. p. 23-31

Página 129 Caderno de Ciências Sociais Aplicadas, Vitória da Conquista/BA, vol. 14, nº 24, ano 14, p. 112-132, jul/dez 2017. 
BOZTEPE, Aysel. Green marketing and its impact on consumer buying behavior. European Journal of Economic and Political Studies, v. 5, n. 1, p. 5-21, 2012.

CARRETE, Lorena; CASTANO, Raquel; FELIX, Reto; CENTENO, Edgar; GONZALEZ, Eva. Green consumer behavior in an emerging economy: confusion, credibility, and compatibility. Journal of Consumer Marketing, v. 29, n.7, p. 470-481, 2012.

CLEVELAND, M., Kalamas, M. and LAROCHE, M. (2005), "Shades of green: linking environmental locus of control and pro-environmental behaviors", Journal of Consumer Marketing, Vol. 22 No. 4, pp. 198-212.

CÔRTES, Pedro Luiz; DO AMARAL MORETTI, Sergio Luiz. Consumo Verde: Um Estudo Transcultural Sobre Crenças, Preocupações e Atitudes Ambientais. Revista Brasileira de Marketing e-ISSN: 2177-5184, v. 12, n. 3, p. 45-76, 2013.

FOLLOWS, Scott B.; JOBBER, David. "ENVIRONMENTALLY RESPONSIBLE PURCHASE BEHAVIOR: a test of a consumer model". European Journal of Marketing, v. 34, n. 5/6, 2000. p. 723-746.

GERSHOFF, Andrew D; FRELS, Judy K. What makes it green? The role of centrality of green attributes in evaluations of the greenness of products. Journal of Marketing, v. 79, n. 1, p. 97-110, 2015.

GONÇALVES-DIAS, S.L.F.; TEODÓSIO, A.S.S.; CARVALHO, S.; SILVA, H.M.R. Consciência ambiental: um estudo exploratório sobre suas implicações para o estudo de Administração. RAEeletrônica, São Paulo, v.8, n.1, art.X, jan./jun. 2009.

GROHMANN, M. Z; BATTISTELlA, L. F.; VELTER, A. N.; CASASOLA, F. Comportamento ecologicamente consciente do consumidor: adaptação da Escala ECCB para o contexto brasileiro. Revista de Gestão Social e Ambiental, v. 6, n. 1, p. 102-116, 2012.

HUBBARD, R.; VETTER, D.; LITTLE, E.; Replication in Strategic Management: Scientific Testing for Validity, Generalizability, and Usefulness. Strategic Management Journal, v. 19, p. 243-254, 1998.

HUNTER, J. The Desperate Need for Replications. Journal of Consumer Research, v. 28, p. 149$158,2001$.

LAGES, N. S.; NETO, A. V. Mensurando a consciência ecológica do consumidor: um estudo realizado na cidade de Porto Alegre. Anais do Encontro da Associação Nacional de Pós-Graduação e Pesquisa em Administração, Salvador, BA, Brasil, 2002.

LAROCHE, Michel; BERGERON, Jasmin; BARBARO-FORLEO, Guido; TOMIUK, MarcAlexandre. Cultural differences in environmental knowledge, attitudes, and behaviours of Canadian consumers. Canadian Journal of Administrative Sciences/Revue Canadienne des Sciences de 1'Administration, v. 19, n. 3, p. 267-282, 2002.

Página 130 Caderno de Ciências Sociais Aplicadas, Vitória da Conquista/BA, vol. 14, nº 24, ano 14, p. 112-132, jul/dez 2017. 
LAROCHE, Michel; TOFFOLI, Roy; KIM, Chankon; MULLER, Thomas.The influence of culture on pro-environmental knowledge, attitudes, and behavior: A Canadian perspective. Advances in Consumer Research, v. 23, p. 196-202, 1996.

MAIBACH, E. Social Marketing for the Environment: Using Information Campaigns to Promote Environmental Awareness and Behavior Change. Health Promotion International. (3) (1993): 209224.

MORRISON, R; MATUSZEK, T.; SELF, D. Preparing a Replication or Update Study in the Business Disciplines. European Journal of Scientific Research, v. 47, n.2, p. 278-287, 2010.

PAÇO, A. M. F.; RAPOSO, M., L. B. Green consumer market segmentation: empirical findings from Portugal. International Journal of Consumer Studies, 34, (2010) pg. 429-436.

PEREIRA PRADO, R., DA SILVA, M., JUNQUEIRA, M., ALMEIDA, L. A Influência do Marketing Verde nos Hábitos de Consumo dos Jovens Universitários dos Cursos de Administração: Estudo em Instituições de Ensino Superior (IES). Revista Brasileira de Marketing e-ISSN: 2177-5184, 10, dec. 2011.

PEREIRA, S. J. N. O Impacto do Argumento Ecológico nas Atitudes dos Consumidores: um estudo experimental. São Paulo. Dissertação de Mestrado, Escola Brasileira de Administração Pública e de Empresas da Fundação Getúlio Vargas, 2003.

POLONSKY, Michael Jay. An introduction to green marketing. Electronic Green Journal, v. 1, n. 2, 1994.

ROBERTS, J.A. and BACON, D.R. (1997), "Exploring the subtle relationships between environmental concern and ecologically conscious consumer behavior", Journal of Business Research, Vol. 40 No. 1, pp. 79-89.

ROBERTS, James A. Green consumers in the 1990s: profile and implications for advertising. Journal of business research, v. 36, n.3, p. 217-231, 1996.

SCHLEGELMILCH, B. B., BOHLEN, G. M. \& DIAMANTOPOULOS, A. (1996), "The link between green purchasing decisions and measures of environmental Consciousness", European Journal of Marketing. 30(5) pp. 35-55.

STRAUGHAN, Robert D.; ROBERTS, James A. Environmental segmentation alternatives: a look at green consumer behavior in the new millennium. Journal of consumer marketing, v. 16, n. 6, p. 558$575,1999$.

TAN, Booi-Chen. The roles of knowledge, threat, and PCE on green purchase behaviour. International Journal of Business and Management, v. 6, n. 12, p. p14, 2011.

TONCAR, M. F.; MUNCH, J. M. Meaningful replication: when is a replication no longer a replication? A rejoinder to Stella and Adam. Journal of Marketing Theory e Practice, v. 18, n. 1, p. 71-80, 2010.

Página 131 Caderno de Ciências Sociais Aplicadas, Vitória da Conquista/BA, vol. 14, n² 24, ano 14, p. 112-132, jul/dez 2017. 


\section{- Cadernos de Clếnclas SOCIAIS APLICADAS}

TSENG, Shih-Chang; HUNG, Shiu-Wan. A framework identifying the gaps between customers' expectations and their perceptions in green products. Journal of cleaner production, v. 59, p. 174184, 2013.

YAM-TANG, Esther PY; CHAN, Ricky YK. Purchasing behaviours and perceptions of environmentally harmful products. Marketing Intelligence \& Planning, v. 16, n. 6, p. 356-362, 1998.

YEH, Tatiana Azevedo. Comportamento de consumo ecologicamente consciente: um estudo comportamento entre Brasil e China. 2015. 71f. Dissertação (Mestrado em Administração de Empresas) - Programa de Pós-Graduação em Administração de Empresas, Fundação Instituto Capixaba de Pesquisas em Contabilidade, Economia e Finanças (FUCAPE), Vitoria, 2015.

ZABKAR, Vesna; HOSTA, Maja. Willingness to act and environmentally conscious consumer behaviour: can prosocial status perceptions help overcome the gap? International Journal of Consumer Studies.37, 3, 257-264, May 2013. ISSN: 14706423.

ZHAO, Hui Hui; GAO, Qian; WU, Yao Ping; WANG, Yuan; ZHU, Xiao Dong. What affects green consumer behavior in China? A case study from Qingdao. Journal of Cleaner Production, v. 63, p. 143-151, 2014. 